\title{
Influence of surface moisture condition on the bond strength to dentin of etch-and-rinse adhesive systems
}

\author{
Eliseu Aldrighi Münchow, Lisia Lorea Valente, Mayara Bossardi, Tanize Cezar Priebe, \\ Cesar Henrique Zanchi, Evandro Piva
}

Universidade Federal de Pelotas - UFPEL, School of Dentistry, Department of Operative Dentistry, Pelotas, RS, Brazil

\begin{abstract}
Aim: To evaluate the immediate microtensile bond strength ( $\mu$ TBS) of three two-step etch-andrinse adhesive systems applied under different dentin surface moisture conditions. Methods: Class V cavities were prepared in seventy-two bovine incisors. Each tooth was randomly allocated into three groups, according to the adhesive system used: Single Bond 2 (SB), Prime \& Bond 2.1 $(\mathrm{PB})$ and $\mathrm{XP}$ Bond $(\mathrm{XPB})$. Each group was divided in three subgroups, according to the dentin moisture condition: over-wet, moist and dry $(n=8)$. For the moist subgroups the adhesive was applied as to the manufacturer's instructions; for the over-wet ones, without drying the cavity after the rinsing procedure; and for the dry subgroups, drying the surface for $20 \mathrm{~s}$. The teeth were restored with Filtek Z-250 and stored in distilled water ( $24 \mathrm{~h}$ ); next, each restoration was sectioned in beam-shaped specimens which were stored for $24 \mathrm{~h}$ at $37^{\circ} \mathrm{C}$. Each specimen was submitted to $\mu$ TBS test (EMIC), and data were analyzed using two-way ANOVA and Tukey test $(p<0.05)$. Results: There was a statistically significant interaction between the adhesive system type and the dentin surface moisture condition ( $\mathrm{p}=0.003)$. SB and XPB presented higher bond strength in the moist dentin condition, and PB showed high $\mu$ TBS values in the dry substrate. Conclusions: The moisture condition influenced the bond strength between the adhesives and dentin. SB performed better in the moist condition, whereas PB and XPB showed satisfactory bond strength in the moist and in the dry substrates. The over-wet dentin condition only impaired bond strength to $\mathrm{SB}$ and $\mathrm{XPB}$.
\end{abstract}

Keywords: tensile strength; solvents; humidity; in vitro.

\section{Introduction}

Differently from the enamel substrate, the adhesive process in dentin is more technique-sensitive, depending on the operator's experience ${ }^{1}$, on the surface moisture conditions of the tooth cavity ${ }^{2-4}$ and also on the substrate morphology $y^{5}$.

Received for publication: May 15, 2014 Accepted: August 08, 2014

Correspondence to:

Eliseu Aldrighi Münchow

Rua Gonçalves Chaves, 457 - CDC-Bio

CEP: 96015-560 Pelotas, RS, Brasil

Phone: +55 $5332226690 / 135$

E-mail: eliseumunchow@hotmail.com Therefore, to promote dentin adhesion, contemporary dental adhesives are categorized into two classes of materials according to the strategy of etching the tooth substrate: the etch-and-rinse or the self-etch techniques. The former uses separate application of an acid solution (commonly phosphoric acid) to etch the surface, whereas the latter uses the adhesive itself ${ }^{6}$. Finished the etching step, a primer and a resin adhesive material should also be applied to complete the adhesive system application.

With regard to the use of etch-and-rinse adhesives in dentin, the acid solution 
is applied for $15 \mathrm{~s}$ followed by rinsing and drying the surface. After that, an ideally moist surface must be achieved to maintain the collagen fibrils in expansion, allowing them to entangle with the resin monomers and to enhance the adhesion process (the so called wet bonding technique) ${ }^{7}$. However, these procedures of rinsing and drying are considered the most critical factors when using etch-andrinse materials; in addition, extensive or complex tooth cavities may present different degrees of wetness, where overwet and dry regions may exist on the same tooth surface ${ }^{8-9}$. As a consequence, that ideally moist surface is difficult to achieve.

Besides the possibility of different moisture degrees remaining in the same tooth cavity after the etching/rinsing/ drying procedures, the adhesion process is also influenced by the adhesive system's composition, mainly the type of solvent used ${ }^{9}$. Among the most common solvents present in dental adhesives there are water, ethanol, acetone and tetrahydrofuran ${ }^{10-11}$. Differently from this pattern, XP Bond ${ }^{\circledR}$ adhesive system (Dentsply Caulk, Milford, MA, USA) ${ }^{12}$, which contains tert-butanol as solvent, is a two-step etch-and-rinse adhesive which is marketed as being a good adhesive agent even in over-wet or dry dentin conditions.

Hence, considering that the solvent plays important roles in the collagen fibrils expansion, in their affinity for the entanglement with the resin monomers, and in the residual water removal of the tooth cavity ${ }^{13}$, the aim of this study was to evaluate the influence of surface moisture condition on the immediate bond strength to dentin of different etchand-rinse adhesive systems. The tested hypothesis was that the adhesives would present different bond strength results at different dentin surface moisture conditions after $24 \mathrm{~h}$ of water storage.

\section{Material and methods}

\section{Tooth preparation and bonding procedures}

Seventy-two bovine incisors were obtained, cleaned and stored in an aqueous $0.5 \%$ solution of Chloramine $\mathrm{T}$ for one week. Class V cavities $(6 \mathrm{~mm}$ length, $4 \mathrm{~mm}$ width, and $2 \mathrm{~mm}$ thickness) were then prepared using diamond bur (FG \#1045, KG Sorensen, Cotia, SP, Brazil) in high-speed handpiece; the bur was replaced by a new one after every 8 teeth prepared. The cavity walls were parallel with each other, as a parallelometer was used. After being prepared, each tooth was randomly allocated in three groups, according to the adhesive system applied: Adper ${ }^{\mathrm{TM}}$ Single Bond 2 (SB); Prime \& Bond 2.1 (PB), and XP Bond (XPB). The brand name, manufacturer, lot number, composition information and application protocol (following manufacturer's instructions) of each adhesive are shown in Table 1. Each group was further divided in three subgroups, according to the dentin surface moisture condition maintained in the cavity: (1) an over-wet condition; (2) a moist condition; or (3) a dry condition $(n=8)$. A $35 \%$ phosphoric acid gel solution (Condac 37; FGM, Joinville, SC, Brazil) was applied on the dentin substrate for $15 \mathrm{~s}$ and rinsed by water-spray for $15 \mathrm{~s}$. The adhesives were then applied (manufacturer's instructions) for all groups (Table 1), except in the over-wet groups where the dentin substrate was maintained plenty of water without surface drying, and in the dried groups where the dentin was thoroughly dried with an air-spray for $20 \mathrm{~s}$. The moist groups where obtained by gently drying the substrate with paper points until a glossy dentin substrate was achieved, according to the wet bonding technique ${ }^{7}$. Next, the adhesives were applied and light-activated for $20 \mathrm{~s}$ using a light-emitting diode (LED) light-curing unit (Radii; SDI, Bayswater, VIC, Australia). The radiance was measured with a digital power meter (Ophir Optronics, Danvers, MA, USA) and it was 900 $\mathrm{mW} / \mathrm{cm}^{2}$. After light-activation, the composite resin (Filtek Z-250, 3M ESPE, St. Paul, MN, USA) was placed in three 2$\mathrm{mm}$-thick increments, which were built up separately using opposite tooth surfaces in an attempt to diminish the C-factor; each increment was then light-activated for $20 \mathrm{~s}$. The restored teeth were stored in distilled water at $37{ }^{\circ} \mathrm{C}$ for $24 \mathrm{hs}$.

\section{Microtensile bond strength ( $\mu$ TBS) test}

The teeth were sectioned longitudinally and transversally using a refrigerated diamond saw at low speed (Isomet 1000; Buehler Ltd, Lake Bluff, IL, USA) to obtain specimens with approximately $0.8 \mathrm{~mm}^{2}$ of transverse-sectional area. The specimens were stored in distilled water at $37{ }^{\circ} \mathrm{C}$ for $24 \mathrm{~h}$ and were fixed with cyanoacrylate adhesive (Super Bonder Gel, Loctite Ltda., São Paulo, SP, Brazil) to a metallic device for the microtensile test ${ }^{14}$. The $\mu$ TBS test was performed in a universal testing machine (DL-500; Emic, São José dos Pinhais, PR, Brazil) at a crosshead speed of $1 \mathrm{~mm} / \mathrm{min}$. Bond strengths were calculated and expressed in $\mathrm{MPa}$, and the premature failures were registered but were not used for the statistical analysis.

\section{Fracture analysis}

The fractured specimens were examined by stereomicroscopy at 40x magnification. The failure patterns were classified as: adhesive at the interface (when the failure occurred at the hybrid layer), cohesive in resin (at the adhesive resin and/or composite), cohesive in dentin, or mixed (when the failure involved different failure patterns).

\section{Statistical analysis}

The statistical program SigmaStat version 3.5 (Systat Software Inc., Chicago, IL, USA) was used for data analysis. The immediate $\mu$ TBS results were submitted to two-way analysis of variance (ANOVA) (adhesive system and dentin moisture condition as factors) and post hoc Tukey's test for multiple comparisons. Statistical significance was established at $5 \%$.

\section{Results}

There was a statistically significant interaction between the adhesive system type and the dentin surface moisture condition $(p=0.003)$. Means (standard deviations) of $\mu$ TBS 
Table 1. Brand names, manufacturers, lot number, composition, and application directions of adhesives.

\begin{tabular}{|c|c|c|c|c|}
\hline Adhesive system & Manufacturer & Lot number & Composition & Application protoc \\
\hline Adper $^{\mathrm{TM}}$ Single Bond 2 (SB) & $\begin{array}{c}\text { 3M ESPE, St. Paul, } \\
\text { MN, USA }\end{array}$ & $9 \times B$ & $\begin{array}{l}\text { Ethanol, water, Bis-GMA, MDP, } \\
\text { HEMA, dimethacrylates, silanated } \\
\text { colloidal, CQ, silica, polyalkenoic } \\
\text { acid copolymer }\end{array}$ & $\begin{array}{l}\text { a (15 s); b; c; d } \\
\quad(2 \text { coats); } ; \\
\quad \text { f (20 s) }\end{array}$ \\
\hline Prime \& Bond $2.1(\mathrm{~PB})$ & $\begin{array}{c}\text { Dentsply Caulk, Milford, } \\
\text { MA, USA }\end{array}$ & 4301070 & $\begin{array}{c}\text { Acetone, Bis-GMA, PENTA, UDMA, } \\
\text { EDAB, photoinitiators butylated } \\
\text { hydroxytoluene, cetylamine } \\
\text { hydrofluoride }\end{array}$ & $\begin{array}{l}\text { a (15 s); b; c; d } \\
\quad(3 \text { coats); e; } \\
\quad \text { f (20 s) }\end{array}$ \\
\hline XP Bond (XPB) & $\begin{array}{c}\text { Dentsply Caulk, Milford, } \\
\text { MA, USA }\end{array}$ & 1105001715 & $\begin{array}{l}\text { T-butanol, PENTA, UDMA, } \\
\text { TEGDMA, HEMA, stabilizers, EDAB, } \\
\text { CQ, functionalized amorphous silica }\end{array}$ & $\begin{array}{l}\text { a (15 s); b; c; d } \\
(1 \text { coat }) ; \text { e }(5 \mathrm{~s}) \\
\quad \mathrm{f}(20 \mathrm{~s})\end{array}$ \\
\hline
\end{tabular}

Abbreviations: Bis-GMA: bisphenol A diglycidyl methacrylate; MDP: 10-10-methacryloyloxydecyl dihydrogen phosphate; HEMA: 2-hydroxyethyl methacrylate; CQ: camphorquinone; PENTA: dipentaerythritol pentacrylate monophosphate; UDMA: urethane dimethacrylate; TEGDMA: tryethyleneglicol dimethacrylate; EDAB: 4-ethyl dimethyl aminobenzoate.

*Procedures according to manufacturer's directions: (a) acid etching; (b) rinse; (c) dry with absorbent paper; (d) apply adhesive; (e) gently air-dry; (f) light curing

are displayed in Table 2. In the over-wet condition, SB demonstrated similar $\mu$ TBS mean to PB $(p=0.308)$, but higher than XPB $(\mathrm{p}<0.001)$, which presented the lowest immediate bond strength mean of this study, but similar to PB $(p=0.441)$. Within the moist dentin condition, SB showed higher bond strength results than the other adhesive systems $(p<0.001)$, which had not differed between each other $(p=0.228)$. In the dry condition, $\mathrm{PB}$ demonstrated similar $\mu$ TBS mean to SB $(p=0.310)$, but higher than XPB $(p=0.008)$, which showed similar bond strength mean to SB $(\mathrm{p}=0.289)$.

Table 2. Immediate microtensile bond strength to dentin (MPa) and standard deviation (SD) presented by the adhesive systems at different dentin surface moisture conditions.

\begin{tabular}{llll}
\hline Adhesive systems & \multicolumn{3}{c}{ Dentin surface moisture condition } \\
& Over-wet & \multicolumn{1}{c}{ Moist } & \multicolumn{1}{c}{ Dry } \\
Single Bond (SB) & ${ }^{A} 25.8(9.1)^{\text {b }}$ & ${ }^{A} 41.1(11.9)^{\text {a }}$ & ${ }^{A B} 25.3(10.3)^{\text {b }}$ \\
Prime \& Bond (PB) & ${ }^{A B} 18.6(10.0)^{\text {b }}$ & ${ }^{B} 27.6(10.4)^{\text {ab }}$ & ${ }^{A} 30.1(7.5)^{\text {a }}$ \\
XP Bond (XPB) & ${ }^{B} 12.7(7.4)^{\text {b }}$ & ${ }^{B} 22.1(10.1)^{\text {a }}$ & ${ }^{B} 19.9(7.6)^{\text {ab }}$ \\
\hline
\end{tabular}

Superscripts capital letters in a same column represent statistically significant differences among the adhesive systems $(p<0.05)$ and superscripts small letters in the same row represent statistically significant differences among dentin conditions $(p<0.05)$.

Figure 1 shows the fracture pattern analysis of the $\mu$ TBS results, where a predominance of adhesive failures has occurred in the over-wet condition; equilibrium of mixed and adhesive failures in the moist dentin surface; and presence of some dentin cohesive failures in the dry condition.

\section{Discussion}

The current investigation selected different types of twostep, etch-and-rinse adhesive systems containing different organic solvents in an attempt to evaluate their influence on the bond strength to dentin in over-wet, moist or dry surface moisture conditions. While Single Bond (SB) is a waterethanol-based adhesive, Prime \& Bond (PB) and XP Bond

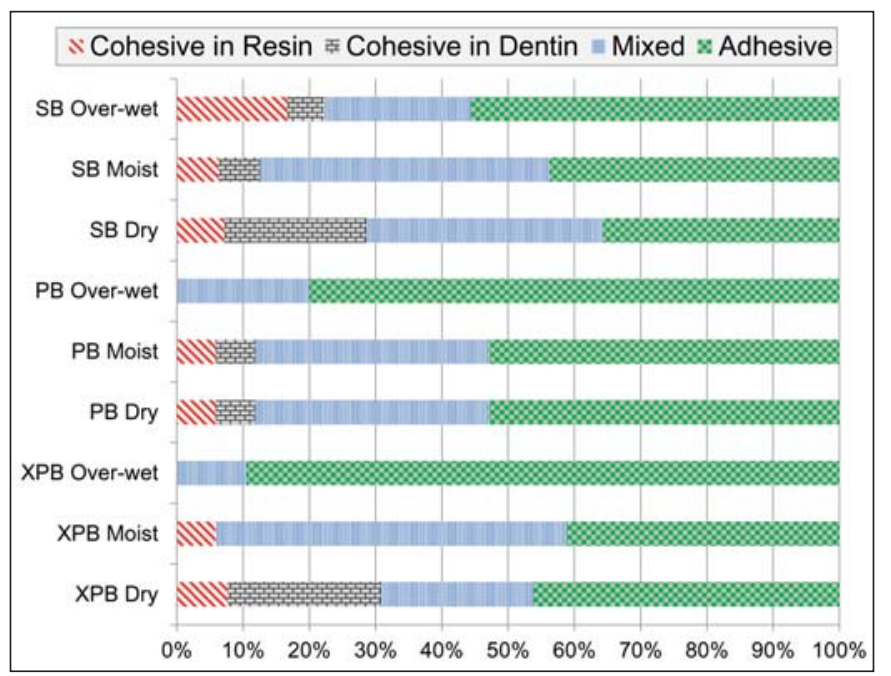

Fig. 1. Fracture pattern (in percentage) of the immediate microtensile bond strength results of the adhesive systems under different dentin surface moisture conditions.

(XPB) are composed by acetone and tert-butanol, respectively. It was hypothesized that the adhesive systems would produce different bond strength results in the different dentin moisture conditions. According to the results displayed in Table 2, this hypothesis can be partially accepted, as SB performed better on the moist dentin substrate, $\mathrm{PB}$ on the dry when compared to over-wet circumstance, and XPB showed higher bond strength in the moist substrate than in the over-wet condition.

The highest bond strength results of the present study were obtained by the application of SB on the moist dentin (Table 2). This material presents a mixture of water and ethanol, which are considered strong hydrogen bonding solvents, although the latter has a higher vapor pressure than the former (43.9 versus $17.5 \mathrm{~mm} \mathrm{Hg}$ at $20^{\circ} \mathrm{C}$, respectively) $)^{15}$, leading to its faster evaporation. Several studies have stated that ethanol-based adhesive systems seem less sensitive to the amount of moisture in dentin ${ }^{16-18}$; nevertheless, this might be true only when a moist dentin is achieved, as the excess 
of water (over-wet groups) or its absence (dry groups) resulted in a significant reduction of the bond strength values. Even though moisture is necessary for a good bonding to dentin, its excess may hamper the water removal, preventing complete monomer infiltration into the demineralized dentin, or even compromising the adhesive polymerization ${ }^{19-20}$. The over-wet moisture condition prepared in the present study, which was obtained without drying the surface after rinsing the tooth cavity, may have probably diluted the adhesive material, turning the hybrid layer porous and more permeable. In the dry surface condition, the over-drying procedure (20 s of airstream application after rinsing) might have collapsed the collagen fibrils, enabling the monomers to properly infiltrate through the collagen matrix ${ }^{11}$. Differently from a waterethanol-based material, $\mathrm{PB}$ is formulated with acetone. According to Reis et al. ${ }^{18}$ (2003) acetone-based adhesive systems require a wetter dentin surface to achieve high bond strengths. Likewise, Cardoso et al. ${ }^{21}$ obtained higher bond strength results in wet dentin when compared to dry dentin. Notwithstanding, da Silva et al. ${ }^{22}$ showed similar bond strengths in both wet and dry substrates when using an acetone-based adhesive. These results corroborate those of the present study, in which higher mean $\mu$ TBS was found in the dry subgroup but without statistically significant difference in comparison with the moist subgroup. A reasonable explanation is that acetone presents high vapor pressure $\left(184 \mathrm{~mm} \mathrm{Hg} \text { at } 20{ }^{\circ} \mathrm{C}\right)^{15}$, which makes it extremely volatile, with fast evaporation from dentin; as a consequence, residual solvent into the adhesive layer is less likely to remain, guaranteeing optimal monomer infiltration/polymerization ${ }^{23}$. Also, complete residual solvent removal increases the quality of adhesives by forming a structure, which is less permeable, more cohesive and densely-packed ${ }^{24}$. On the other hand, PB applied on the over-wet dentin condition resulted in significantly lower $\mu$ TBS mean than the value obtained in the dry condition. Acetone, which presents lower polarity than water and ethanol, does not form hydrogen bonds, thus it would not be able to interact easily with over-wet dentin. Consequently, residual water may have been entrapped within the fibrils, enabling the resin diffusion and subsequent polymerization $^{11}$.

Despite the water-ethanol and acetone-based adhesive systems being more common in dentistry, XPB contains tertbutanol as the organic solvent. It has been claimed to be less technique-sensitive, due to its improved ability of diffusing through collagen fibrils, even in a collapsed state ${ }^{12}$. Thus, it was supposed to be a good bonding agent option to be applied in some adverse conditions such as over-wet or over-dry dentins. According to an in vivo clinical evaluation, XPB met the criteria for a provisional acceptance (American Dental Association guidelines), presenting less than 5\% failure rate after 6 months of clinical performance ${ }^{25}$. Additionally, other studies have demonstrated satisfactory bond strength results when using this adhesive 26-27. Nevertheless, the present study results showed that XPB was associated with the lowest $\mu$ TBS values in all the three surface conditions evaluated (Table 2), although similar bond strength means for PB (in the over-wet and moist conditions) and for SB (in the dry condition) could be observed. A possible reason for this result is the lower vapor pressure of tert-butanol (26 $\mathrm{mm} \mathrm{Hg}$ at $\left.20{ }^{\circ} \mathrm{C}\right)^{28}$ when compared to ethanol and acetone (43.9 and $184 \mathrm{~mm} \mathrm{Hg}$ at $20{ }^{\circ} \mathrm{C}$, respectively), hampering its ability to remove the residual water molecules and its own evaporation from the dentin surface. Another possible explanation is that monomers such as HEMA can decrease the vapor pressure of water, interfering with the removal of any residual water ${ }^{29}$, and considering that XPB contains HEMA (Table 1), the tert-butanol ability of controlling dentin wetness may have been even more reduced.

All the afore-mentioned different bond strength results are in accordance with the failure patterns obtained in this study (Figure 1). The highest $\mu$ TBS means were obtained in the moist dentin condition, which has shown a balance of adhesive and mixed failures for all the adhesive systems applied. On the other hand, in the dry condition, which may have reduced the cohesive strength of the dentin substrate, a predominance of adhesive failures has occurred plus the appearance of some dentin cohesive failures. Differently, the over-wet dentin condition was marked by a predominance of adhesive failures, suggesting that the hybrid layer was at least in some extent defective and poorly produced.

Within the limitations of the present study, it may be concluded that the moisture condition influenced the microtensile bond strength to dentin among the evaluated adhesive systems. Single Bond 2 performed better in the moist condition, whereas Prime \& Bond and XP Bond showed adequate bond strength in the moist and dry substrates. Notwithstanding, the over-wet dentin condition only significantly impaired the bond strength of the Single Bond 2 and XP Bond adhesive systems.

\section{Acknowledgements}

The authors acknowledge the Brazilian National Council for Scientific and Technological Development (CNPq) for supporting the present study (grant 156232/2010-3) and CAPES/MEC (Brazilian Government) for scholarship.

\section{References}

1. Unlu N, Gunal S, Ulker M, Ozer F, Blatz MB. Influence of operator experience on in vitro bond strength of dentin adhesives. J Adhes Dent. 2012; 14: 223-7.

2. Jacquot B, Durand JC, Farge P, Valcarcel J, Deville de Periere D, Cuisinier F. Influence of temperature and relative humidity on dentin and enamel bonding: a critical review of the literature. Part 1. Laboratory studies. J Adhes Dent. 2012; 14: 433-46.

3. Plasmans PJ, Creugers NH, Hermsen RJ, Vrijhoef MM. The influence of absolute humidity on shear bond adhesion. J Dent. 1996; 24: 425-8.

4. Pupo YM, Michel MD, Gomes OM, Lepienski CM, Gomes JC. Effect of the regional variability of dentinal substrate and modes of application of adhesive systems on the mechanical properties of the adhesive layer. J Conserv Dent. 2012; 15: 132-6.

5. Cavalcanti AN, de Souza ES, Lopes GS, de Freitas AP, de Araújo RPC, Mathias $P$. Effect of a desensitizing dentifrice on the bond strength of different adhesive systems. Braz J Oral Sci. 2013; 12: 148-52. 
6. Pashley DH, Tay FR, Breschi L, Tjaderhane L, Carvalho RM, Carrilho M, et al. State of the art etch-and-rinse adhesives. Dent Mater. 2011; 27: 1-16.

7. Kanca J, 3rd. Improving bond strength through acid etching of dentin and bonding to wet dentin surfaces. J Am Dent Assoc. 1992; 123: 35-43.

8. Miyazaki M, Onose $\mathrm{H}$, Moore BK. Effect of operator variability on dentin bond strength of two-step bonding systems. Am J Dent. 2000; 13: 101-4.

9. Tay FR, GwinnettAJ, Wei SH. The overwet phenomenon: a transmission electron microscopic study of surface moisture in the acid-conditioned, resin-dentin interface. Am J Dent. 1996; 9: 161-6.

10. Fontes ST, Ogliari FA, Lima GS, Bueno M, Schneider LF, Piva E. Tetrahydrofuran as alternative solvent in dental adhesive systems. Dent Mater. 2009; 25: 1503-8.

11. Manso AP, Marquezini L Jr, Silva SM, Pashley DH, Tay FR, Carvalho RM. Stability of wet versus dry bonding with different solvent-based adhesives. Dent Mater. 2008; 24: 476-82.

12. Dentsply XP BOND for eXtra Performance. Scientific Compendium. Konstanz: Dentsply DeTrey; 2006.

13. Koliniotou-Koumpia E, Kouros P, Koumpia E, Helvatzoglou-Antoniades M. Shear bond strength of a "solvent-free" adhesive versus contemporary adhesive systems. Braz J Oral Sci. 2014; 13: 64-9.

14. Münchow EA, Bossardi M, Priebe TC, Valente LL, Zanchi $\mathrm{CH}$, Ogliari FA, et al. Microtensile versus microshear bond strength between dental adhesives and the dentin substrate. Int J Adhes Adhes. 2013; 46: 95-9.

15. Gallo JR, Burgess JO, Xu X. Effect of delayed application on shear bond strength of four fifth-generation bonding systems. Oper Dent. 2001; 26: 48-51.

16. Finger WJ, Balkenhol M. Practitioner variability effects on dentin bonding with an acetone-based one-bottle adhesive. JAdhes Dent. 1999; 1: 311-4.

17. Jacobsen T, Soderholm KJ. Effect of primer solvent, primer agitation, and dentin dryness on shear bond strength to dentin. Am J Dent. 1998; 11: 225-8.

18. Reis A, Loguercio AD, Azevedo CL, de Carvalho RM, da Julio Singer M, Grande RH. Moisture spectrum of demineralized dentin for adhesive systems with different solvent bases. J Adhes Dent. 2003; 5: 183-92.

19. Spencer $P$, Wang $Y$. Adhesive phase separation at the dentin interface under wet bonding conditions. J Biomed Mater Res. 2002; 62: 447-56.

20. Tay FR, Gwinnett JA, Wei SH. Micromorphological spectrum from overdrying to overwetting acid-conditioned dentin in water-free acetonebased, single-bottle primer/adhesives. Dent Mater. 1996; 12: 236-44.

21. Cardoso P de C, Lopes GC, Vieira LC, Baratieri LN. Effect of solvent type on microtensile bond strength of a total-etch one-bottle adhesive system to moist or dry dentin. Oper Dent. 2005; 30: 376-81.

22. da Silva MA, Rangel PM, Barcellos DC, Pagani C, Rocha Gomes Torres C. Bond strength of adhesive systems with different solvents to dry and wet dentin. J Contemp Dent Pract. 2013; 14: 9-13.

23. Bail M, Malacarne-Zanon J, Silva SM, Anauate-Netto A, Nascimento FD, Amore R, et al. Effect of air-drying on the solvent evaporation, degree of conversion and water sorption/solubility of dental adhesive models. J Mater Sci Mater Med. 2012; 23: 629-38.

24. Ye Q, Spencer P, Wang Y, Misra A. Relationship of solvent to the photopolymerization process, properties, and structure in model dentin adhesives. J Biomed Mater Res A. 2007; 80: 342-50.

25. Blunck U, Knitter K, Jahn KR. Six-month clinical evaluation of XP BOND in noncarious cervical lesions. J Adhes Dent .2007; 9(Suppl 2): 265-8.

26. Hegde M, Manjunath J. Bond strength of newer dentin bonding agents in different clinical situations. Oper Dent. 2011. Jun 24. [Epub ahead of print].

27. Margvelashvili M, Goracci C, Beloica M, Papacchini F, Ferrari M. In vitro evaluation of bonding effectiveness to dentin of all-in-one adhesives. J Dent. 2010; 38: 106-12.

28. Teagarden DL, Baker DS. Practical aspects of lyophilization using nonaqueous co-solvent systems. Eur J Pharm Sci. 2002; 15: 115-33.

29. Takahashi M, Nakajima M, Hosaka K, Ikeda M, Foxton RM, Tagami J. Long-term evaluation of water sorption and ultimate tensile strength of HEMA-containing/-free one-step self-etch adhesives. J Dent. 2011; 39: 506-12. 